\title{
Tailoring Carbon Nanostructure for High Frequency Supercapacitor Operation
}

\author{
Pritesh Hiralal, ${ }^{1}$ Gemma Rius, ${ }^{2}$ Piers Andrew, ${ }^{3}$ \\ Masamichi Yoshimura, ${ }^{4}$ and Gehan A. J. Amaratunga ${ }^{1}$ \\ ${ }^{1}$ Electrical Engineering Division, University of Cambridge, $9 \mathrm{~J}$. J. Thomson Avenue, Cambridge CB3 OFA, UK \\ ${ }^{2}$ Nagoya Institute of Technology, Gokiso, Showa, Nagoya 466-8555, Japan \\ ${ }^{3}$ Nokia Research Center, Broers Building, 21 J. J. Thomson Avenue, Cambridge CB3 OFA, UK \\ ${ }^{4}$ Toyota Technological Institute, 2-12-1 Hisakata, Tempaku, Nagoya 468-8511, Japan
}

Correspondence should be addressed to Pritesh Hiralal; pritesh.hiralal@cantab.net

Received 2 February 2014; Accepted 13 March 2014; Published 8 May 2014

Academic Editor: Matthew T. Cole

Copyright (C) 2014 Pritesh Hiralal et al. This is an open access article distributed under the Creative Commons Attribution License, which permits unrestricted use, distribution, and reproduction in any medium, provided the original work is properly cited.

\begin{abstract}
The possibility of enhancing the frequency performance of electrochemical capacitors by tailoring the nanostructure of the carbon electrode to increase electrolyte permeability is demonstrated. Highly porous, vertically oriented carbon electrodes which are in direct electrical contact with the metallic current collector are produced via MPECVD growth on metal foils. The resulting structure has a capacitance and frequency performance between that of an electrolytic capacitor and an electrochemical capacitor. Fully packaged devices are produced on $\mathrm{Ni}$ and $\mathrm{Cu}$ current collectors and performance compared to state-of-the-art electrochemical capacitors and electrolytic capacitors. The extension of capacitive behavior to the AC regime $(\sim 100 \mathrm{~Hz})$ opens up an avenue for a number of new applications where physical volume of the capacitor may be significantly reduced.
\end{abstract}

\section{Introduction}

Electrical energy storage devices exploit a range of charge storage mechanisms, ranging from purely physical processes, as employed in dielectric capacitors, all the way to the full electrochemical reactions employed in batteries. These different mechanisms result in remarkable performance variation between the various technologies.

Dielectric capacitors store energy in the electric field arising from polarization of highly mobile surface charges. These devices have the capability of high frequency operation, but their overall energy storage density is small. Batteries, on the other hand, store chemical potential energy and require ions to move through an electrolyte as well as undergo chemical reactions at the electrodes. This results in considerably slower charge and discharge rates, but since the bulk of the electrode is used, the resulting energy density is several orders of magnitude higher. Various electrical energy storage devices, for example, electrolytic capacitors, supercapacitors [1], and radical batteries [2], sit between these extremes and have correspondingly intermediate performances. For example, supercapacitors have a battery-like structure, consisting of electrodes and an electrolyte, but store charge in an electric field like a dielectric capacitor [1].

Carbon is the material of choice for most electrochemical capacitors due to its low cost, abundance, rich chemistry, and electrochemical stability. Its multiple allotropes and plethora of morphologies have made it possible to tailor carbon for multiple applications such as electrodes which are transparent [3], have high surface area [4], have high conductivity [5], and are flexible [6] or even stretchable [7]. Commercial supercapacitor electrodes typically consist of activated carbon with highly developed specific surface areas $\left(\sim 2000 \mathrm{~m}^{2} / \mathrm{g}\right)$ and intricate pore structures [8]. However, this very characteristic, which provides high capacitance values, also limits the ionic permeability through the electrodes, which, in turn, results in slower charge/discharge times. As a consequence, their frequency of operation is limited to a few Hertz, thereby excluding any AC applications. 
In this work, the morphology of the carbon electrode material is tailored for high electrolyte permeability and easy pore access with the aim of increasing the possible frequency range of operation. In particular, we focus on carbon nanowalls (CNWs), a form of 2D carbon grown vertically onto a metallic current collector. The application of CNWs for high frequency supercapacitors builds up on the concept demonstrated by Miller et al. [9]. As the synthesis requires no use of a catalytic layer, it results in direct electronic contact of the CNWs to the collector as well as excellent electrolyte permeability. Pioneering efforts on CNW synthesis have been made by the Wu group [10]. CNWs intrinsic large specific surface area makes them attractive for charge storage applications, but also for other electrochemical applications such as sensing [11]. A high density of edges means they have been considered as field emission sources [12] as well as templates for the growth of other nanostructured materials [13].

\section{Experimental Method}

Direct growth of vertically oriented CNWs onto various substrates by microwave plasma enhanced chemical vapor deposition (MPECVD) has already been described elsewhere $[14,15]$. The synthesis is driven by field-boosted mecha$\operatorname{nism}(\mathrm{s})$, and the result is advantageous as it generally consists of single step processing with very reasonable deposition rates [16]. Plasma deposition systems are often scalable, opening an avenue for larger scale applications. Flexible substrates such as metal foils or wires can be used. Here, a MPECVD system (CVD-CN-100, Ulvac, Japan Ltd.) is used for CNW growth on metallic foils ( $\mathrm{Ni}$ and $\mathrm{Cu}$, Nilaco Corp., 99.9\% purity).

In brief, the CNW deposition is conducted within a vertical cylindrical quartz tube about $5 \mathrm{~cm}$ in diameter and $24 \mathrm{~cm}$ in length, using a coupled rectangular waveguide to deliver microwaves from a microwave source $(2.45 \mathrm{GHz}$, $500 \mathrm{~W}$ ), applied perpendicular to the tube. Two circular parallel plate electrodes are used to apply a DC bias along the longitudinal axis of the reactor. Temperature increase inside the reactor is a result of microwave field energy absorption and energy transfer from the establishment of an electrical current between the two electrodes. No additional heater or temperature regulation is available. The microwave field and applied bias voltage cause the ionization of either annealing $\left(\mathrm{H}_{2}\right)$ or growth/reaction gases $\left(\mathrm{H}_{2}+\mathrm{CH}_{4}\right)$ and establish a vertically directed electrical current.

As electron mobility is higher than ion mobility, the formation of a positive ion plasma sheath upon the lower electrode that holds the sample is understood, which under certain conditions can lead to carbon material deposition onto the target substrate. For CNT deposition, the directionality of the gas species in combination with suitable catalytic particles, resulting from the decomposition of thin metal layers, for example, enables the creation of CNT nucleation sites and allows the formation of vertically aligned CNTs [17]. CNW formation, on the other hand, is not driven by the presence of catalytic nanoparticles/materials; however an alumina spacer is used to electrically isolate the target sample from the lower electrode. When $\mathrm{Ni}$ substrates are used, the electrically insulating spacer prevents the formation of carbon fibres or tubes [18].

The growth conditions used are common for both $\mathrm{Ni}$ and $\mathrm{Cu}$ foil samples presented here. The process starts with 3 minutes $\mathrm{H}_{2}$ flow $(20 \mathrm{sscm}$ ), during which the bias voltage is progressively increased from 0 to $100 \mathrm{~V}$. After sample annealing, reaction gases, $\mathrm{H}_{2}: \mathrm{CH}_{4}(20: 20 \mathrm{sccm})$, are introduced for 10 minutes, in which the first 2 minutes are a transitory stage while the DC bias is steadily raised from $100 \mathrm{~V}$ to $200 \mathrm{~V}$. A pressure of $1.5 \mathrm{~m}$ Torr is kept constant during both processing phases. Under these conditions we obtain a uniform black, densely covered surface with vertically oriented graphene flakes forming a porous nanostructured 3D architecture (Figure 1). In the literature plasma-deposited carbon flakes are typically made from single layer graphene sheets to a few-tens-of-nanometers-thick graphite.

As a comparison, electrodes were also fabricated using state-of-the-art activated carbons designed for lower resistance values (Kuraray, YP-80). An aqueous paste was prepared by blending water, $1.6 \%$ wt carboxymethyl cellulose (CMC) solution, binder (styrene-butadiene), and activated carbon powder $(9.3: 5: 0.2: 5)$. The resulting paste was then bar-coated onto a $1 \mathrm{mil}$ aluminum foil with conducting carbon coating (Intelicoat Technologies) and dried overnight in a vacuum oven at $80^{\circ} \mathrm{C}$, resulting in a $\sim 30 \mu \mathrm{m}$ thick film. Samples of the correct size where then punched out for capacitor fabrication.

Packaged electrochemical capacitors are fabricated by sandwiching two $\sim 1 \mathrm{~cm}^{2}$ as-grown electrodes between $25 \mu \mathrm{m}$ thick cellulose separators soaked in a solution of $1 \mathrm{M}$ tetraethyl ammonium tetrafluoroborate $\left(\mathrm{TEABF}_{4}\right)$ in propylene carbonate. The sandwich structure was then packaged into type 2032 coin cells. Despite the slightly high internal resistance which results from this specific type of packaging, it is useful to demonstrate the practical performance figures on a packaged device. Electrochemical properties of the capacitor were studied with a potentiostat/galvanostat (Autolab PGSTAT 302N).

\section{Results and Discussion}

The typical microscopic morphology of CNWs is shown in Figure 1(a). Vertically oriented and curly flakes are randomly, but homogeneously, distributed on the Ni sample surface. Similar morphologies are obtained on $\mathrm{Cu}$ foil substrates (not shown) $[14,15]$. Flake dimensions are about $1 \mu \mathrm{m}$ or less in length and thicknesses around $10-20 \mathrm{~nm}$, thinning from base to top. The structure leads to pores of 100-400 nm aperture.

Raman spectra (532 nm laser excitation) of the deposited material show it to be crystalline and multilayered (graphitized) carbon with limited crystal size and with presence of defects (Figure 1(b)). G and 2D bands are found at wavenumbers 1586 and $2690 \mathrm{~cm}^{-1}$, respectively, as a signature of graphite-like material (in-plane vibrational modes) [19]. The $2 \mathrm{D}$ peak width $\left(\mathrm{FWHM} \sim 83 \mathrm{~cm}^{-1}\right.$ ) indicates that the material consists of multilayer graphene, although precision in the number of layer determination is compromised, specifically, by the CNW vertical orientation. Additionally, the relative intensities of the $\mathrm{G}$ and $2 \mathrm{D}$ bands are dominated by the 

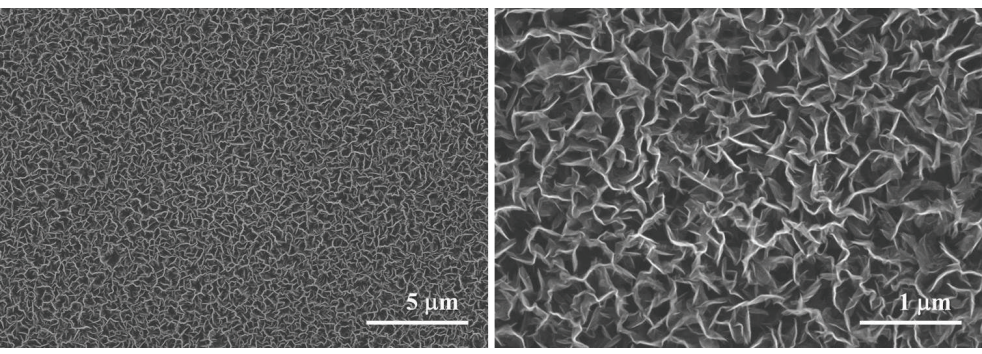

(a)

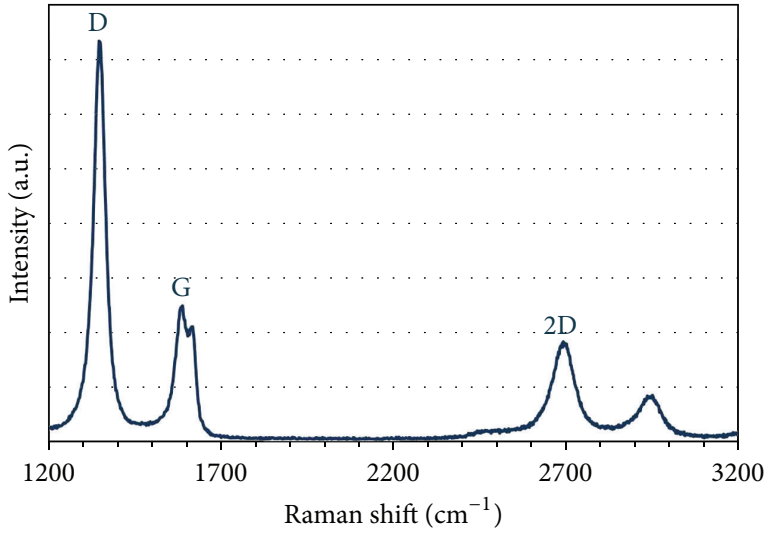

(b)
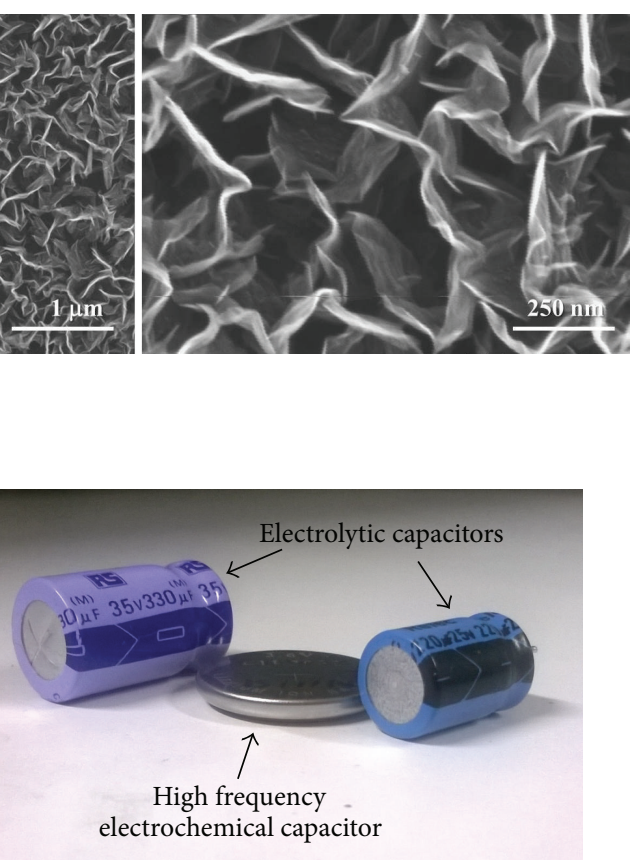

(c)

FIgURE 1: Building blocks of the electrochemical capacitors (ECs) of the current study. (a) Top view scanning electron microscopy (SEM) images of CNWs deposited onto Ni foil by MPECVD. (b) Typical Raman spectrum of CNWs on Ni substrate. (c) Size comparison of the CNW EC and electrolytic capacitors of similar capacitance. It should be noted here that the active volume of the device within the coin cell is only $\sim 0.01 \mathrm{~cm}^{3}$ and a much smaller coin cell could be used while still maintaining performance.

strong(er) disorder-induced D band $\left(1348 \mathrm{~cm}^{-1}\right)[20]$. The similarity of these spectra with that of MPECVD multiwalled CNTs [18] should be noted.

In summary, the Raman spectroscopy analysis confirms the observations of CNWs by SEM imaging (roughness, possibility of amorphous carbon covering the CNWs surface, high density of edges, etc.). Grain boundaries and limited crystal domains (finite size) are characteristic features of CNWs. Further characterization, such as by XPS and HRTEM, would be needed to assist a refined elucidation of chemical and structural characteristics of CNWs as well as details of their morphological variability: purity, contamination, thinning from top to base, contact to the metal substrate and its characteristics, and so forth.

The packaged coin cell is shown in Figure 1(c), along with commercial electrolytic capacitors of similar capacitance. The 2032 coin cell packaging is used as standard in our lab, and although a significant part of it remains hollow in this case, already a significant volume reduction can be observed for similar capacitance values. This potential size reduction is advantageous for multiple applications. It is estimated that packaged volumes can easily reach $\sim 1 / 10$ th the volume of the equivalent electrolytic capacitor, but this depends very much on the frequency to be used as well as the type of packaging chosen. However, it is important to note that applications of electrochemical capacitors (ECs) will be more limited as a result of the smaller voltage window $(\sim 2.5 \mathrm{~V})$.
Cyclic voltammograms of the four devices on study are shown on Figure 2. At slow scan rates, up to $\sim 0.1 \mathrm{~V} / \mathrm{s}$, the voltammogram of the activated carbon device (Figure 2(a)) has an almost rectangular (ideal) form common in these sorts of devices and a capacitance $\sim 2$ orders of magnitude higher than the other devices in the comparison. The latter is expected due to the higher mass of carbon in each electrode ( 2 versus $\ll 0.2 \mathrm{mg} / \mathrm{cm}^{2}$ ). However, note that as scan rate goes up, the resistive component starts dominating and the curves tend towards a resistance-dominated behavior. Figures 2(b) and 2(c) show the voltammograms for CNWs on $\mathrm{Cu}$ and $\mathrm{Ni}$, respectively. The rectangular shape is well maintained even at $50 \mathrm{~V} / \mathrm{s}$, already indicating better capacitive behavior at higher frequencies. It should be noted that, despite using carbon electrodes with an organic electrolyte (propylene carbonate, $\mathrm{PC}$ ), the voltage window is limited to around 1-1.1 V. This seems to occur even when using more electrochemically stable current collectors such as aluminium. The reason behind this is not yet clear and is subject to further study. Figure 2(d) shows measured voltammograms of a commercial electrolytic capacitor of similar capacitance, illustrating near-ideal behaviour.

Several things may be extracted from the cyclic voltammograms. Figure 3(a) shows the variation of current at midpoint of the cycle with scan rate for the different devices. Values are normalised with respect to the highest value of each sample. Note that, given the similarity between the CNW 


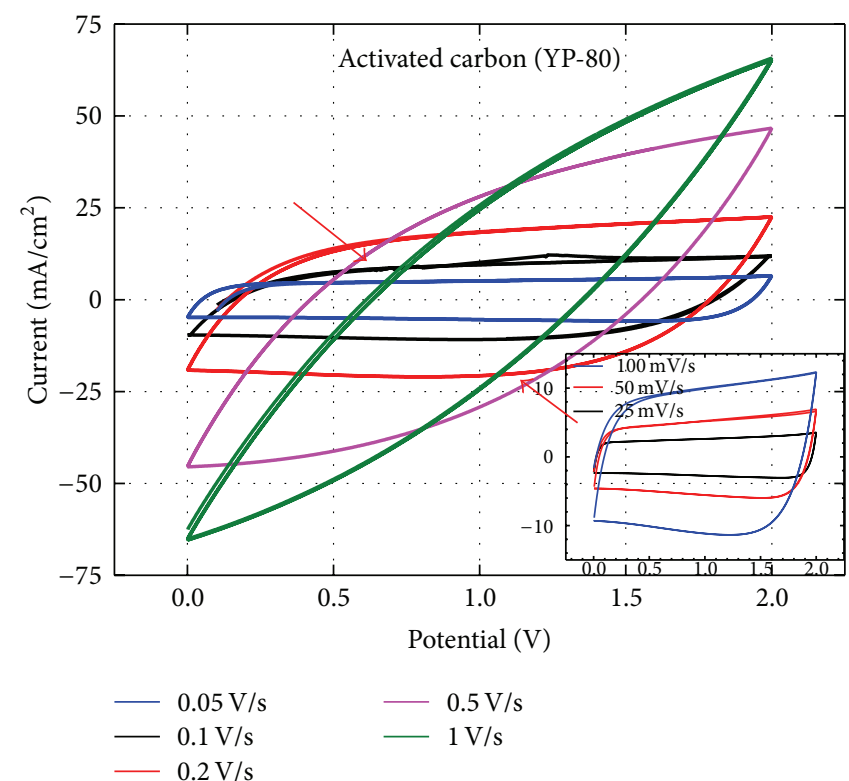

(a)

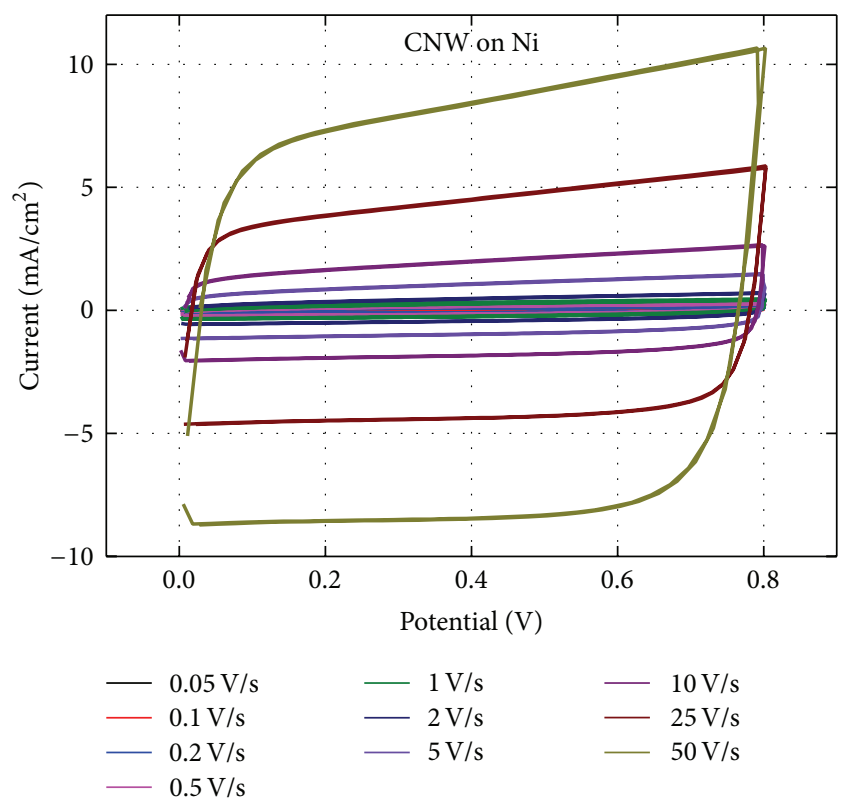

(c)

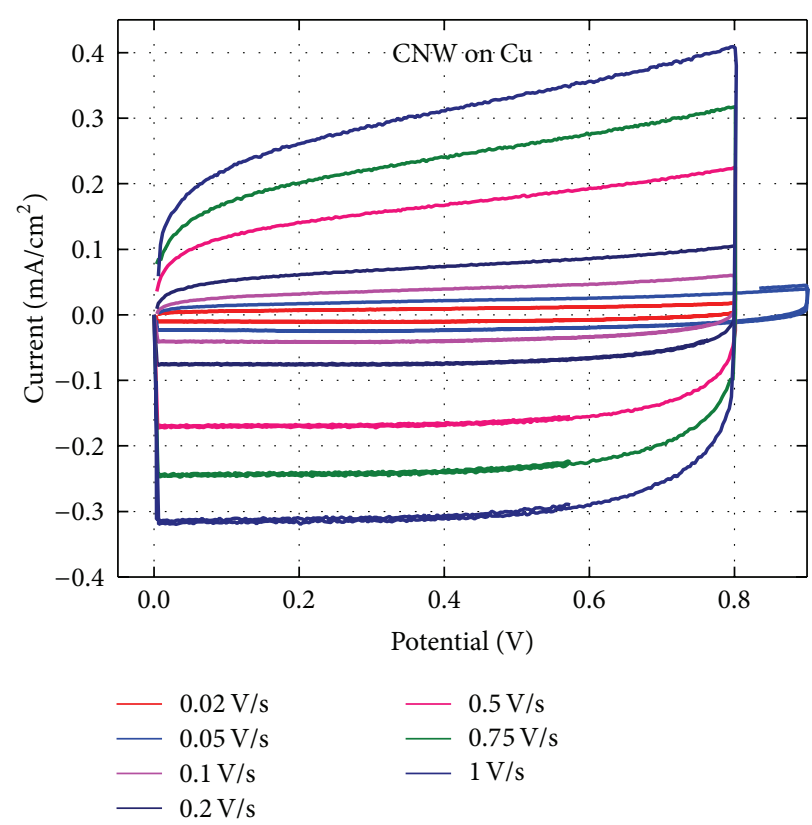

(b)

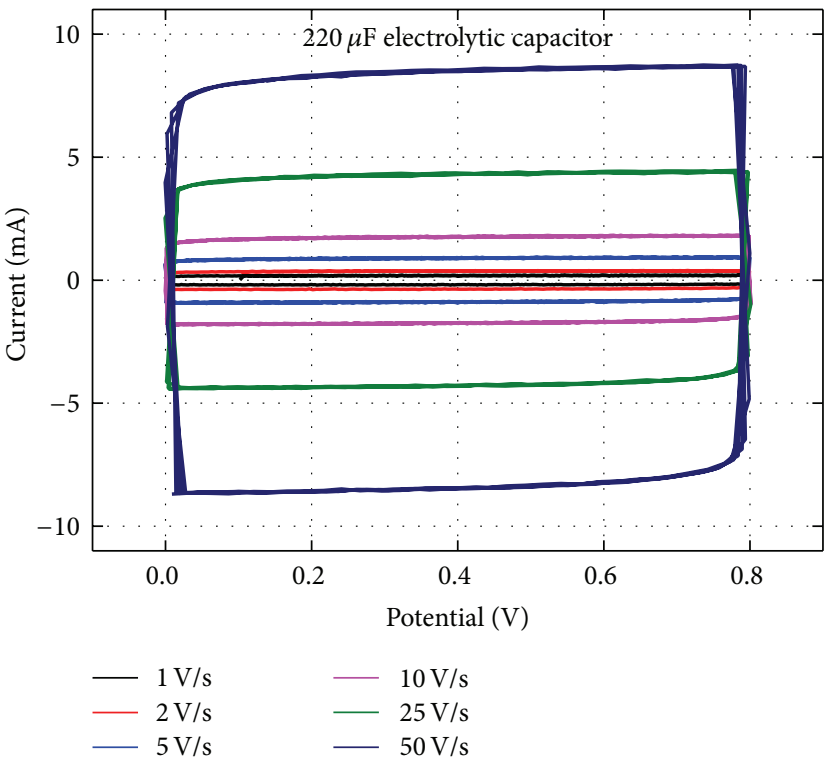

(d)

Figure 2: Cyclic voltammograms of the devices under study: (a) activated carbon electrodes (YP-80), inset: zoomed version of the slow scan rates showing the rectangular shape, (b) CNW on Cu electrodes, (c) CNW on Ni electrodes, and (d) a commercial $220 \mu \mathrm{F}$ electrolytic capacitor. Note the approximately 2 orders of magnitude difference in the current density of the activated carbon device and the rest, but also how the curves from this device tend toward a pure resistance with increasing scan rate.

samples, only the device on $\mathrm{Ni}$ is shown for the sake of clarity. A linear relation signifies constant capacitance with cycling rate which implies all the surface area of the electrode is easily accessible by the electrolyte at a fast rate, that is, high permeability. Although over the faster scan rates the CNW device exhibits a quasilinear relation, which has also been noted with graphene electrodes [21], there is a nonlinearity on the slower scan rates, better observed in Figure 3(b) which shows the normalized capacitance versus scan rate. Here an initial decay can be observed, after which capacitance remains relatively constant. This would suggest the presence of small pores which are not so readily accessible at faster scan rates, but also that there is in addition a significant proportion of easily accessible pores giving the constant capacitance at higher scan rates. This is not the case with activated carbon, where pore structure is hierarchical, from macropores to 


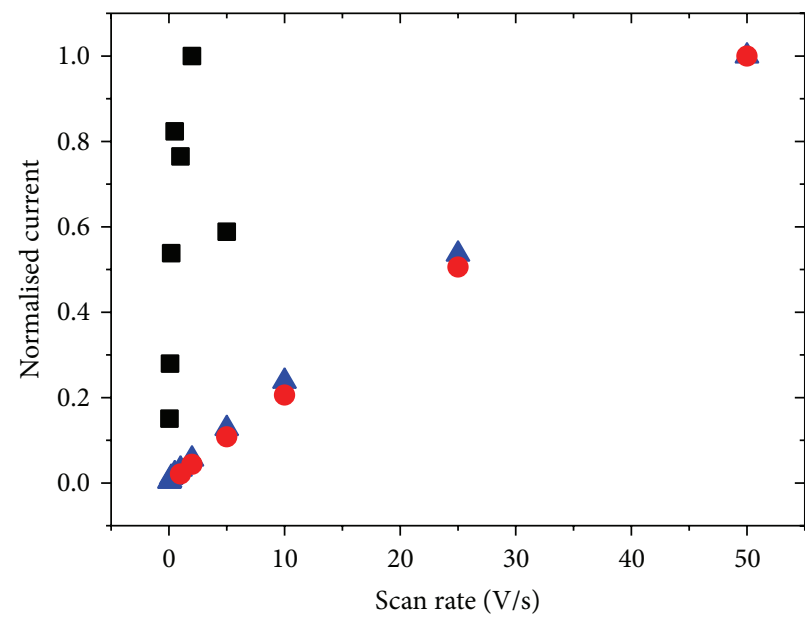

- CNW on Ni EC

- Activated carbon EC

- Electrolytic capacitor

(a)

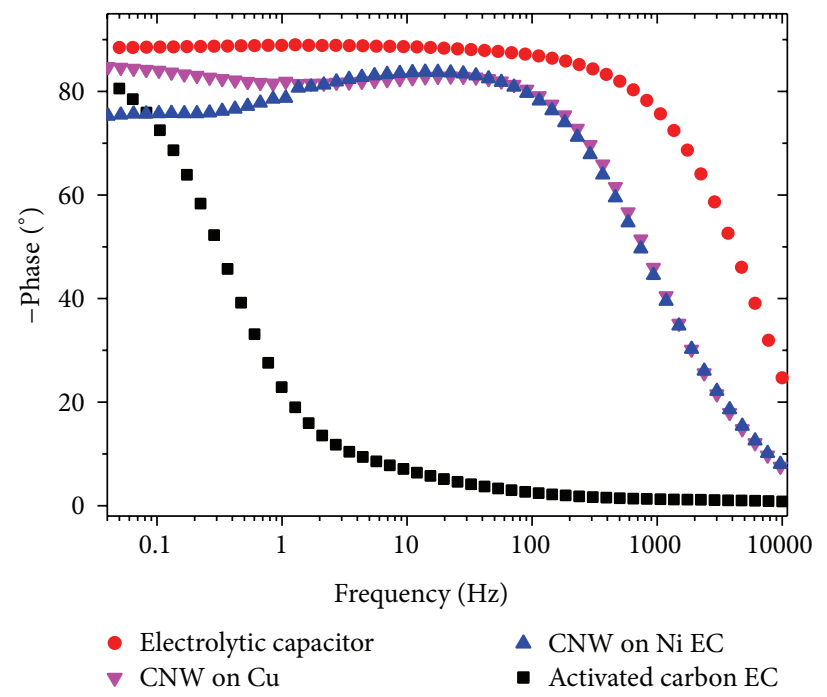

(c)

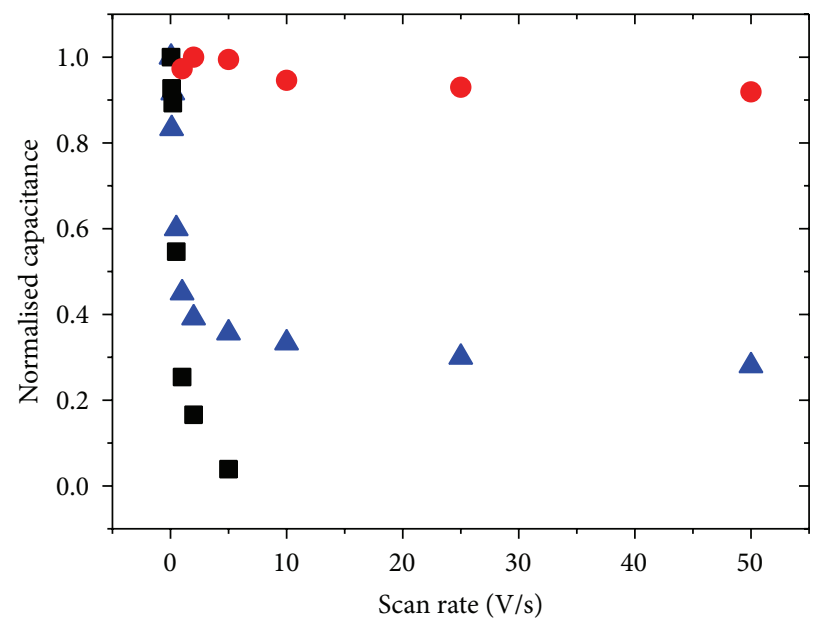

- CNW on Ni EC

- Activated carbon EC

- Electrolytic capacitor

(b)

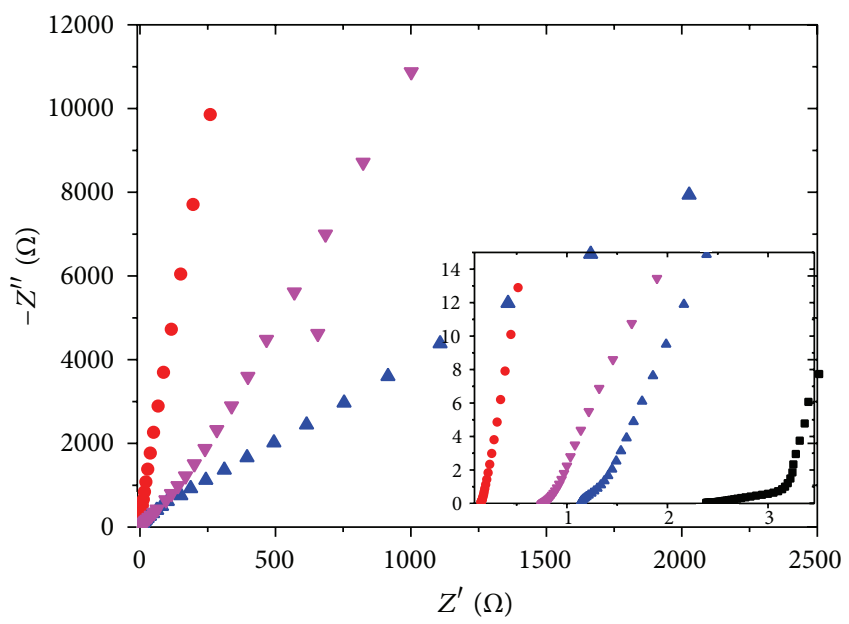

- Activated carbon EC

- Electrolytic capacitor

$\Delta \mathrm{CNW}$ on Ni EC

$\checkmark \mathrm{CNW}$ on $\mathrm{Cu}$

(d)

FIGURE 3: Comparative rate and frequency performance of the capacitors. (a) Normalised current (from CVs) versus scan rate. (b) Normalised capacitance versus scan rate (from CVs) showing the rate of decay of capacitance with increasing scan rates. (c) Bode phase plot. (d) Electrochemical impedance spectroscopy data expressed as Nyquist plots over the frequency range of $10 \mathrm{kHz}-0.05 \mathrm{~Hz}$. Inset shows zoom at low impedance.

mesopores to micropores, each with increased resistance to electrolyte penetration [8]. A significant contribution to the large surface area of activated carbon is provided by the micropores $(<2 \mathrm{~nm})$, and as a result capacitance fades very quickly with scan rate. The electrolytic capacitor on the other hand maintains constant capacitance throughout the tested range. It should be noted that the absolute capacitance of the electrolytic capacitor is of similar magnitude to the CNW capacitor at higher frequencies, whilst still retaining a size advantage. However, it can be noticed from Figure 3(b) that at low scan rates the capacitance of the CNW capacitor goes up to $\sim 3 \mathrm{x}$ the value at fast scan rates. Peak capacitances at
$(50 \mathrm{mV} / \mathrm{s})$ are $0.6,205$, and $0.18 \mathrm{mF}$ for the CNW, activated carbon, and electrolytic device, respectively.

Figure 3(c) shows a Bode phase plot of the capacitors studied. All capacitors show capacitive behavior (near $-90^{\circ}$ phase angle) at low frequency and resistive behavior (near $0^{\circ}$ phase angle) at high frequency. However, the transition occurs below $1 \mathrm{~Hz}$ for the activated carbon device, whilst the CNW capacitors start changing above $100 \mathrm{~Hz}$. The electrolytic capacitor tested decayed at around $1000 \mathrm{~Hz}$. Again, this disparity can be ascribed to electrode porosity differences. Figure 3(d) shows the impedance spectra of the devices, which were measured from frequencies ranging from $10 \mathrm{kHz}$ 
to $0.05 \mathrm{~Hz}$. The inset shows the high frequency and low impedance area in detail. Note that the activated carbon sample is only visible in the expanded view as the impedance values only span a small range. The positioning of these spectra in the inset is a clear indication of the relative device equivalent series resistances (ESR). There are no features associated with a series passive layer (high-frequency semicircle), which would add series resistance. At high frequencies $(10 \mathrm{kHz})$, supercapacitors behave like a resistance. At low frequency, the imaginary part of the impedance sharply increases and the plot tends to a vertical line characteristic of capacitive behavior (note the relative scales of the axes) [22]. The relative angle with respect to the real axis, or in other words the rate of increase of real impedance, provides an indication of the degree of resistance of the system; that is, the $\mathrm{Cu}$ based device is less resistive than the $\mathrm{Ni}$ one.

\section{Conclusions}

The possibility of enhancing the frequency performance of electrochemical capacitors by tailoring the nanostructure of the carbon electrode to increase electrolyte permeability is demonstrated. Highly porous carbon electrodes which are in direct electrical contact with the metallic current collector are produced via MPECVD growth on metal foils. The resulting structure has a capacitance and frequency performance between that of an electrolytic capacitor and an electrochemical capacitor. However, the extension of capacitive behavior to the $\mathrm{AC}$ regime $(\sim 100 \mathrm{~Hz})$ opens up an avenue for a number of new applications where physical volume of the capacitor may be significantly reduced.

We demonstrate the performance of fully packaged devices and compare performance on $\mathrm{Ni}$ and $\mathrm{Cu}$ current collectors. Although the capacitance, frequency performance, and nanostructure are not significantly different between the different current collectors, for low ESR applications the $\mathrm{Cu}$ electrode is preferred, although this may limit the voltage window.

\section{Conflict of Interests}

The authors declare that there is no conflict of interests regarding the publication of this paper.

\section{Acknowledgments}

This work was supported under the Nokia-Cambridge Strategic Alliance in Nanoscience and Nanotechnology and Japan National Nanotechnology Platform Initiative.

\section{References}

[1] B. E. Conway, Electrochemical Supercapacitors: Scientific Fundamentals and Technological Applications, Springer, 1999.

[2] K. Nakahara, S. Iwasa, M. Satoh et al., "Rechargeable batteries with organic radical cathodes," Chemical Physics Letters, vol. 359, no. 5-6, pp. 351-354, 2002.

[3] P. Hiralal, H. Wang, H. E. Unalan et al., "Enhanced supercapacitors from hierarchical carbon nanotube and nanohorn architectures," Journal of Materials Chemistry, vol. 21, no. 44, pp. 17810-17815, 2011.

[4] O. Barbieri, M. Hahn, A. Herzog, and R. Kötz, "Capacitance limits of high surface area activated carbons for double layer capacitors," Carbon, vol. 43, no. 6, pp. 1303-1310, 2005.

[5] M. Cole, P. Hiralal, K. Ying et al., "Dry-transfer of aligned multiwalled carbon nanotubes for flexible transparent thin films," Journal of Nanomaterials, vol. 2012, Article ID 272960, 8 pages, 2012.

[6] H. Y. Jung, M. B. Karimi, M. G. Hahm, P. M. Ajayan, and Y. J. Jung, "Transparent, flexible supercapacitors from nanoengineered carbon films," Scientific Reports, vol. 2, article 773, 2012.

[7] P. Hiralal, S. Haque, D. Cotton, and C. Bower, "A stretchable electronic apparatus and associated methods," WO2013128068 A106, 2013.

[8] P. Simon and Y. Gogotsi, "Materials for electrochemical capacitors," Nature Materials, vol. 7, no. 11, pp. 845-854, 2008.

[9] J. R. Miller, R. A. Outlaw, and B. C. Holloway, "Graphene double-layer capacitor with ac line-filtering performance," Science, vol. 329, no. 5999, pp. 1637-1639, 2010.

[10] Y. Wu, P. Qiao, T. Chong, and Z. Shen, "Carbon nanowalls grown by microwave plasma enhanced chemical vapor deposition," Advanced Materials, vol. 14, no. 1, pp. 64-67, 2002.

[11] E. Luais, M. Boujtita, A. Gohier et al., "Carbon nanowalls as material for electrochemical transducers," Applied Physics Letters, vol. 95, no. 1, Article ID 014104, 2009.

[12] A. T. H. Chuang, J. Robertson, B. O. Boskovic, and K. K. K. Koziol, "Three-dimensional carbon nanowall structures," Applied Physics Letters, vol. 90, no. 12, Article ID 123107, 2007.

[13] B. Yang, Y. Wu, B. Zong, and Z. Shen, "Electrochemical synthesis and characterization of magnetic nanoparticles on carbon nanowall templates," Nano Letters, vol. 2, no. 7, pp. 751-754, 2002.

[14] G. Rius and M. Yoshimura, "Synthesis control for carbon nanowalls on copper supports pro development of green energy applications," E-Journal of Surface Science and Nanotechnology, vol. 10, pp. 305-309, 2012.

[15] G. Rius and M. Yoshimura, "Structured nanocarbon on various metal foils by microwave plasma enhanced chemical vapor deposition," Journal of Physics: Conference Series, vol. 417, no. 1, Article ID 012010, 2013.

[16] A. T. H. Chuang, B. O. Boskovic, and J. Robertson, "Freestanding carbon nanowalls by microwave plasma-enhanced chemical vapour deposition," Diamond and Related Materials, vol. 15, no. 4-8, pp. 1103-1106, 2006.

[17] G. Rius, I. T. Clark, and M. Yoshimura, "Robust operation and performance of integrated carbon nanotubes atomic force microscopy probes," Journal of Physics: Conference Series, vol. 417, no. 1, Article ID 012072, 2013.

[18] I. T. Clark, G. Rius, Y. Matsuoka, and M. Yoshimura, "Mechanical and electronic characteristics of scanning probe microscopy probes based on coaxial palladium nanowire/carbon nanotube hybrid structures," Journal of Vacuum Science and Technology B: Microelectronics and Nanometer Structures, vol. 28, no. 6, pp. 1148-1152, 2010.

[19] M. S. Dresselhaus, A. Jorio, M. Hofmann, G. Dresselhaus, and R. Saito, "Perspectives on carbon nanotubes and graphene Raman spectroscopy," Nano Letters, vol. 10, no. 3, pp. 751-758, 2010.

[20] Z. H. Ni, H. M. Fan, Y. P. Feng, Z. X. Shen, B. J. Yang, and Y. H. $\mathrm{Wu}$, "Raman spectroscopic investigation of carbon nanowalls," 
Journal of Chemical Physics, vol. 124, no. 20, Article ID 204703, 2006.

[21] K. Sheng, Y. Sun, C. Li, W. Yuan, and G. Shi, "Ultrahigh-rate supercapacitors based on eletrochemically reduced graphene oxide for ac line-filtering," Scientific Reports, vol. 2, article 247, 2012.

[22] P. L. Taberna, P. Simon, and J. F. Fauvarque, "Electrochemical characteristics and impedance spectroscopy studies of carboncarbon supercapacitors," Journal of the Electrochemical Society, vol. 150, no. 3, pp. A292-A300, 2003. 

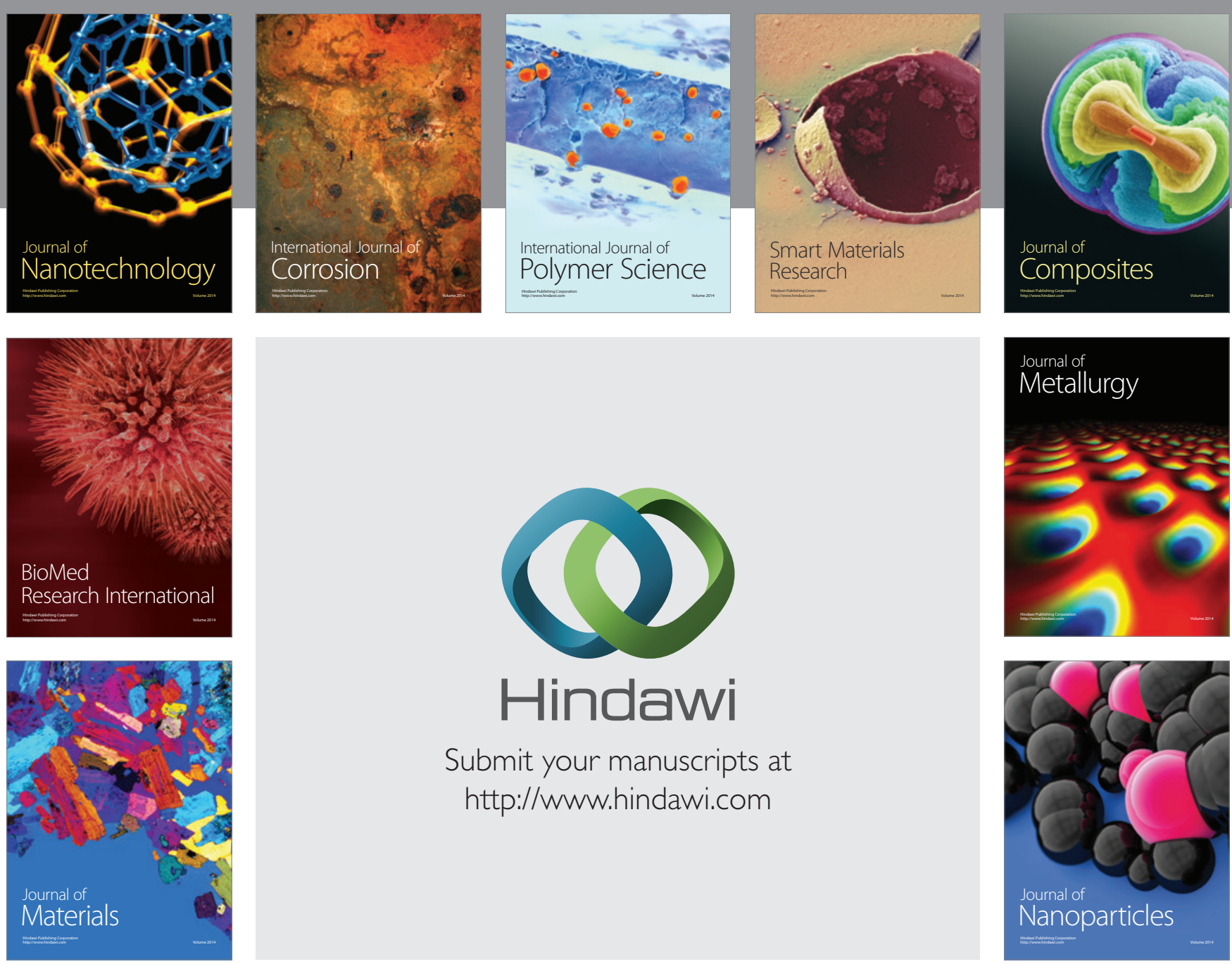

Submit your manuscripts at http://www.hindawi.com
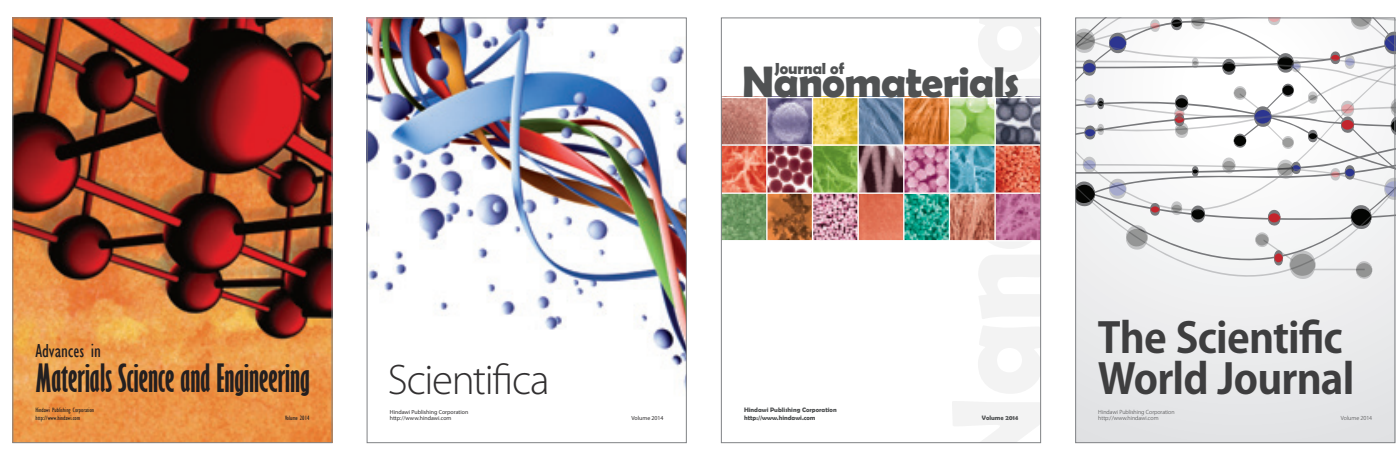

\section{The Scientific World Journal}
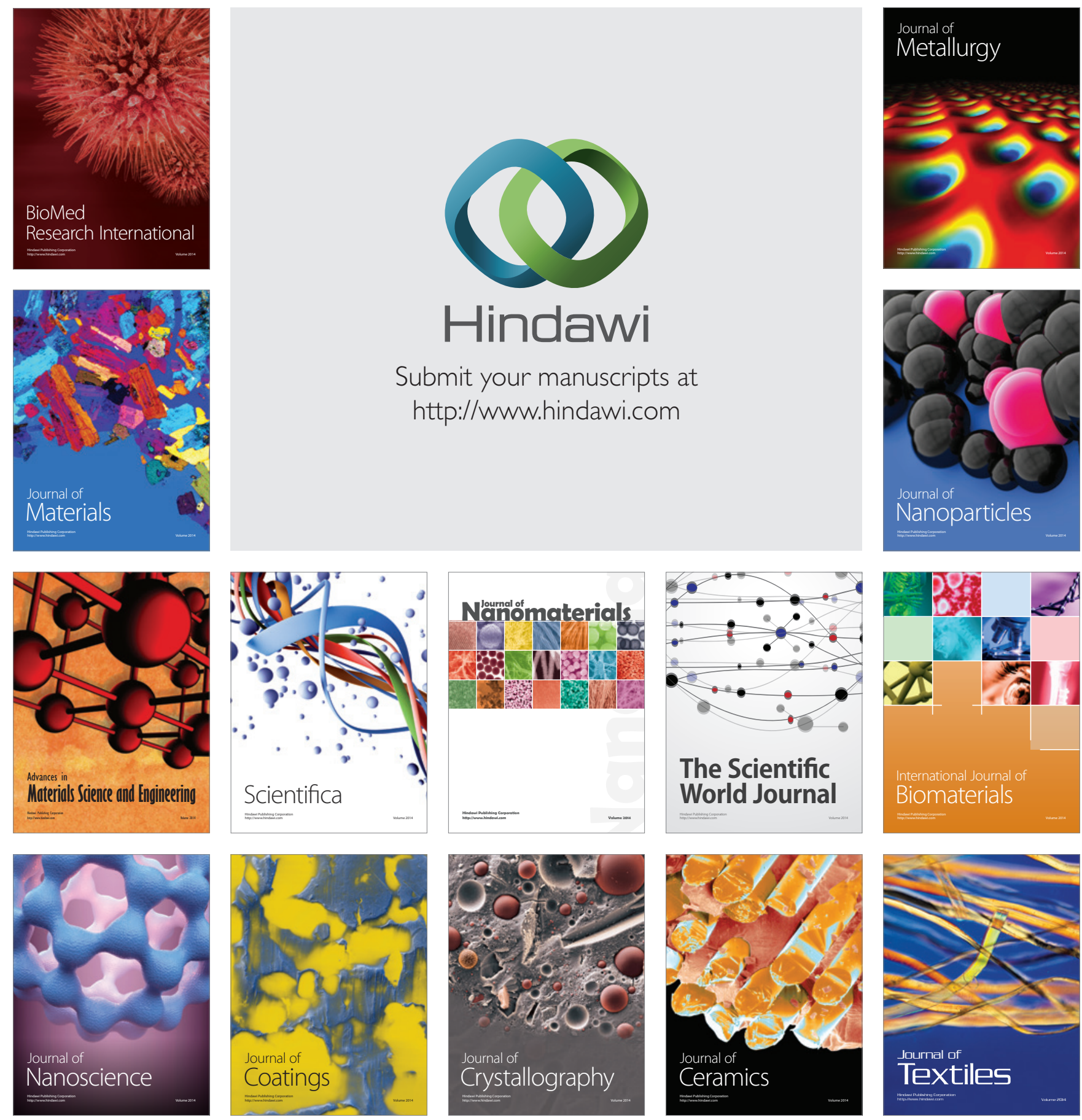